\title{
DATA: Digital Archiving and Transformed Analytics
}

\author{
Sheldon Liang, Peter McCarthy, Melanie Van Stry \\ Department of Computer Science, Division of STEM, Lane College, Jackson, TN, USA \\ Email: SLiang@lanecollege.edu, PMcCarthy@lanecollege.edu, MVanstry@lanecollege.edu
}

How to cite this paper: Liang, S., McCarthy, P. and Van Stry, M. (2021) DATA: Digital Archiving and Transformed Analytics. Intelligent Information Management, 13, 70-95.

https://doi.org/10.4236/iim.2021.131004

Received: November 3, 2020

Accepted: January 26, 2021

Published: January 29, 2021

Copyright (c) 2021 by author(s) and Scientific Research Publishing Inc. This work is licensed under the Creative Commons Attribution International License (CC BY 4.0).

http://creativecommons.org/licenses/by/4.0/

(c) (i) Open Access

\begin{abstract}
As cloud service becomes more and more capable, available and powerful, wiseCIO has emerged from an innovative roadmap toward archival Content Management Service (aCMS) and massive Content Delivery Service (mCDS) in support of Anything-as-a-Service (XaaS) via Digital Archiving and Transformed Analytics (DATA); DATA aims to automate UBC with FAST solutions throughout a feasible, analytical, scalable and testable approach. This paper, based on the novel wiseCIO (web-based intelligent service engaging Cloud Intelligence Outlet), presents digital archiving and transformed analytics via machine learning automata for intelligent UBC processes to liaise with Universal interface for human-computer interaction, enable Brewing aggregation (differing from traditional web browsing), and engage Centered user experience. As one of the most practical aspects of artificial intelligence, machine learning is applied to analytical model building and massive and/or multidimensional Online Analytical Processing (mOLAP) for more intelligent cloud service with little explicit coding required. DATA is central to useful information via archival transformation and analytics, and utilizable intelligence for Business, Education and Entertainment (iBEE) in support of decision-making. As a result, DATA orchestrates wiseCIO to promote ACTiVE XaaS that enables accessibility, contextuality and traceability of information for vast engagement with various cloud services, such as aCMS (archival Content Management Service), COSA (Context-Oriented Screening Aggregation), DASH (Deliveries Assembled for fast Search and Hits), OLAS (Online Learning via Analytical Synthesis), REAP (Rapid Extension and Active Presentation), and SPOT (Special Points On Top) with great ease.
\end{abstract}

\section{Keywords}

wiseCIO: Web-Based Intelligent Service Engaging Cloud Intelligence Outlet, LIAiSE: Layouts of Interactivity and Actionability via Intelligent Systems Engineering, iDEA: Intelligence-Driven Efficient Automation, ACTiVE: 
Accessible/Available, Contextual and Traceable Information for Vast

Engagement, winCOM: Web-Intensive Composite

\section{Introduction}

wiseCIO [1] takes a leadership toward the "Anything as a Service" or XaaS era that enhances organizational cloud service experience without building their own data-centers, and maintains Information Technology personnel [2].

DATA is dedicated to XaaS by orchestrating wiseCIO to provide various cloud services, such as PaaS (a Platform for Development) and SaaS (a Software as a Service) by introducing such conceptual models as digital archiving via transformed analytics [3] [4] [5] [6], efficient automation via machine 1 earning automaton [7] [8] [9], universal liaise, brewing aggregation and centered engagement against traditional web content management and delivery [10].

\subsection{DATA Innovates Cloud Service}

wiseCIO innovates cloud service transitioning from "exhausted-ness" model to "excelled-ness" model [1] over DATA that liaises with universal interface, enables brewing aggregation, and engages centered user experience against traditional web service with following versus':

Unfriendly vs Universal - the design of user interface (UI) aims to liaise with client and server $(\mathrm{C} / \mathrm{S})$ in either unfriendly LIAR (layout of interface for action and reaction) or universal LIAiSE (layout of interactivity and actionability via intelligent systems engineering). LIAR reflects the "ad hoc" UI design while LIAiSE with universal interface design automated with little coding required.

Browsing vs Brewing - web browsing is to view web content by simply retrieving formatted documents from the remote server, downloaded onto the client's device, then overlapping the current context, which may cause pages often-swapping to distract the user due to lacking of logically-interrelated content. Brewing aggregation, innovated by wiseCIO, analyze/synthesize and then transform digitally archived documents into useful information for decisionmaking, and usable intelligence applied to business, education and entertainment (iBEE).

Chasing vs Centered-traditional websites are organized in plain web pages so that user experience (UX) while surfing may become awkward without a centralized context, "like a chasing after wind (webpages)" without centralized context. So chasing UX seems AWK (aimless \& wonder knocking). Centered UX represents user engagement of ACT with accessibility, contextuality and traceability via ubiquitous service, load-balancing and failover over DATA.

\subsection{DATA Involves FAST Solutions}

Digital archiving helps to transform computational thinking into manageable 
processes so that archival content management and massive content delivery [11] [12] [13] are made possible throughout a FAST approach as follows:

- Feasible decomposition represents a manageable process of breaking a big problem into smaller problems and archiving them to hold their very own part of the whole.

- Analytical pattern recognition reflects an automated process of analyzing and looking for a repeating sequence for "brewing" aggregation via reusable retrieval and assembly.

- Scalable abstraction denotes an extracting and synthesizing process of removing unnecessary parts of a problem and creating a general solution to a family of problems via an agile and bidirectional approach: top-down analysis and bottom-up synthesis.

- Testable algorithms express a sequential and concurrent process of step-bystep instructions to solve a primary problem, then other problems without needing additional solutions.

FAST approach is feasible, analytical, scalable and testable to assist computational thinking from "all in mind" to automata of machine learning that is reflected on manageable processing, and automated by intelligent UBC processes.

\subsection{Major Contributions and Organization}

wiseCIO has emerged from an innovative roadmap toward XaaS to aid (automate-involve-dedicate) UBC involving archival content management (aCMS) and massive content delivery (mCDS) throughout a FAST approach. Major contributions are accountable as follows:

Universal interface automates LIAiSE (with human-computer interaction) for user interface design through feasible layouts of interactivity and actionability via intelligent systems engineering.

Brewing aggregation involves archival content management and massive content delivery, instead of simply retrieving, into useful and utilizable intelligence for business, education and entertainment (iBEE) in support of decision-making.

Centered experience dedicates ACTiVE XaaS over DATA with accessibility, contextuality and traceability of information for vast engagement with centered user experience in order for efficiency and usability of the developed system in use [14].

The rest of the paper is organized as follows:

- Section 2 LIAiSE with computational thinking.

- Section 3 iDEA for brewing aggregation

- Section 4 ACTiVE XaaS in use

- Section 5 Conclusion: DATA in transitioning

\section{LIAiSE with Computational Thinking}

Intelligent systems for engineering [15] promote an interdisciplinary approach on how to engineer, implement and manage complex systems over their life 
cycles. As discussed in [1], wiseCIO enables computational thinking and manageable processing via DATA (digital archiving and transformed analytics) represents the applied intelligence to the multi-perspective UBC approach as illustrated in Figure 1.

Figure 1 brings out a blueprint of applying computational thinking to DATA (Digital Archiving and Transformed Analytics) to engineer wiseCIO by automating UBC throughout LIAiSE with universal interface, iDEA for brewing aggregation, and ACTiVE with XaaS.

\subsection{Feasible Decomposition for Web-Intensive Composites}

In terms of archival content management [16], as discussed in the introduction, web-intensive composite (winCOM) has been innovated as essential archiving components for content presentation, aggregation, and delivery. The feasible decomposition, taking Miami University as an example, represents breakdowns of a "giant" institution (seemingly like a big bite we can't chew) into possible winCOM that are smaller and easy to manage feasibly.

Figure 2 illustrates one of the thumb-ups of computational thinking is its

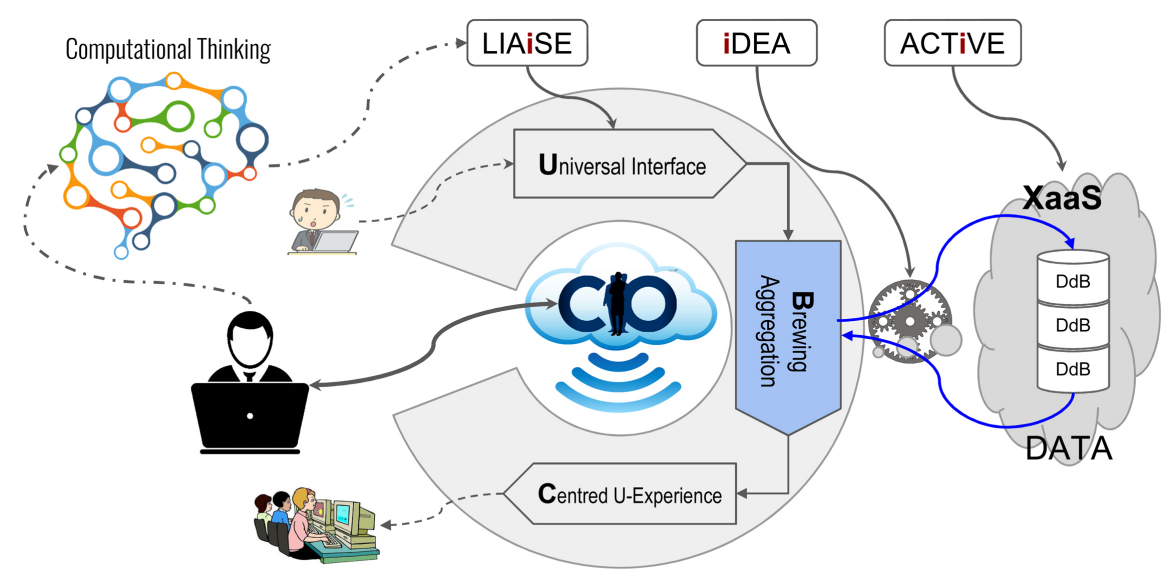

UBC-automated wiseCIO for XaaS

Figure 1. Computational thinking applied throughout LIAiSE, iDEA, and ACTiVE.

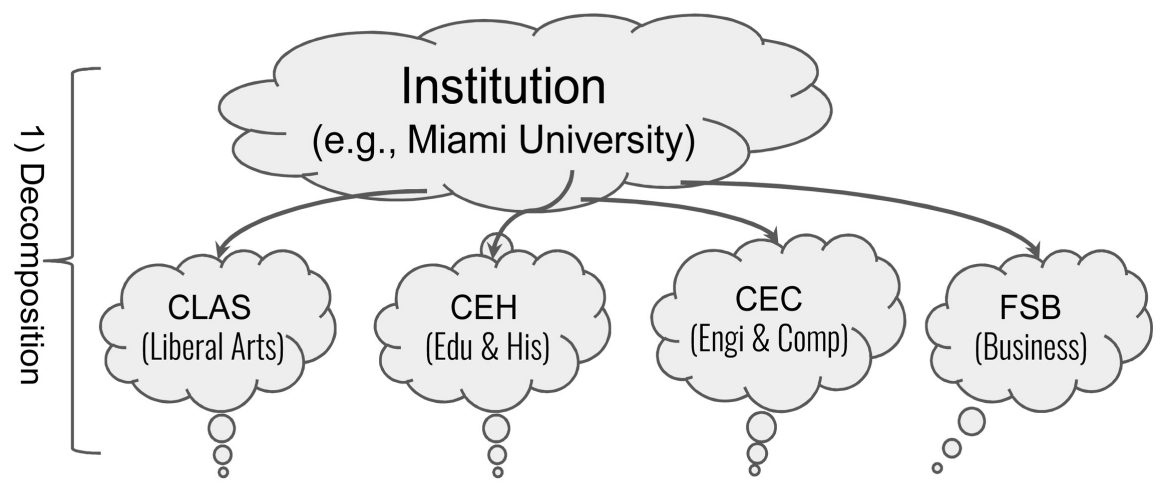

Figure 2. Archival digitalization starts with feasible decomposition in principle of "divide-conquer". 
feasibility to break down a large university into separate colleges. If the college is still too large to handle, the breakdown process continues until easy enough to handle.

The decompositional feasibility reflects an idea in engineering DATA to manage archival content interrelating consistencies and logical similarities so that a "college" should be highly cohesive (more interactions within) and lowly coupling (less ties to outside). Web intensiveness involving interactivity for human-computer interaction and actionability via interoperating between the client and the server (C/S interoperation) is embodied as winCOM of QAS features (queryable, assemblable \& synthesizable) for logical organization and relational information groupings in support of the principle of "divide-conquer" for manageability and computability [17].

\subsection{Analytical Pattern Recognition for Iterative Sequence}

The winCOM through feasible decompositions does not treat the divided parts mysteriously, but meaningfully as analyzed with the aim at analytical parts that are identified and recognized [18] for a repeating sequence that is reusable for similar problems.

For instance, Miami University has a couple of colleges underneath, so an applicable "repeating sequence" represents the organizational similarities of personnel and administration in general: a dean, associate dean, an assistant to officials, offices and academic committee, where winCOM enables a work in progress from a "cloud" (unclear) to a "concrete" (integrally digitalized) component, as illustrated in Figure 3.

Figure 3 indicates that winCOM applies the similar strategy applied to reusable patterns via winCOM that are recognized through computational thinking and manageable processing (CTMP) from a "cloudy" concept (of an educational institution), to a concrete modeling solution.

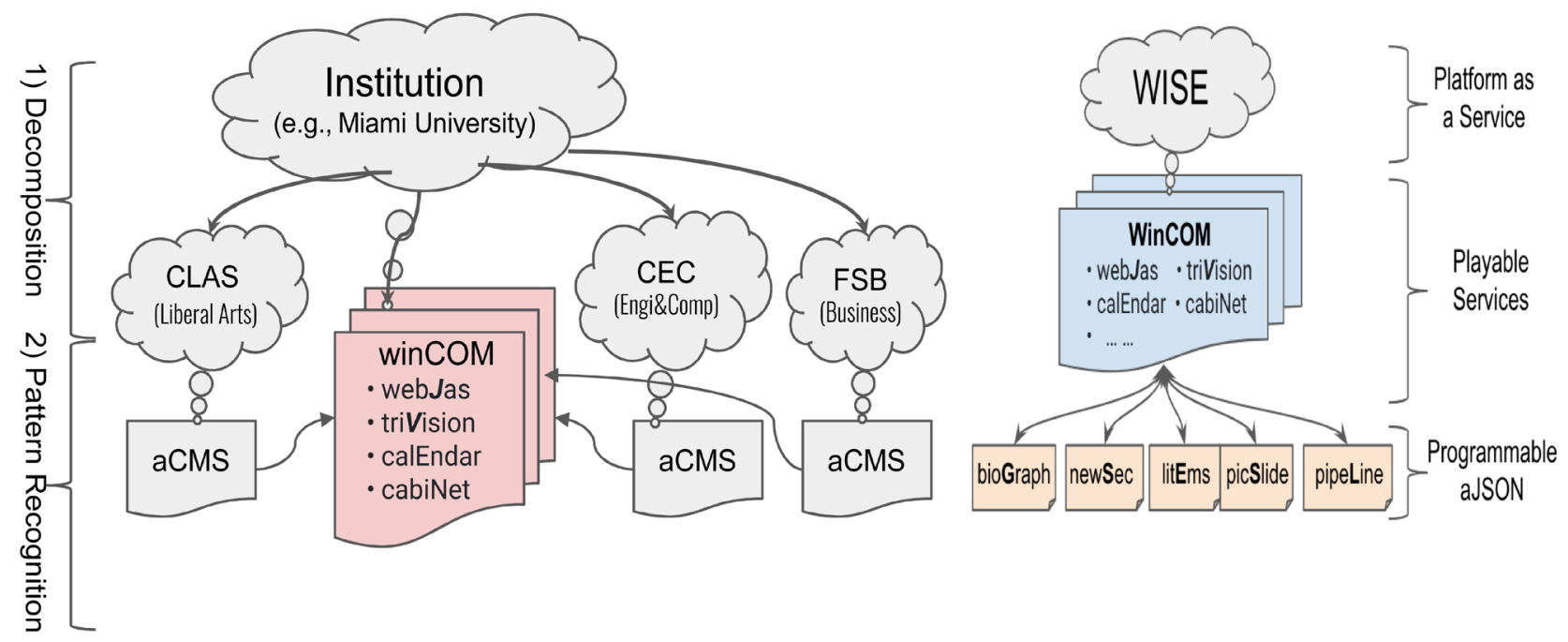

Figure 3. Archival digitalization and transformation through analytical pattern recognition. 
Apparently, archival content management of some "well-done work" is embodied as a repeating sequence over all colleges through archival transformations and analytics via winCOM and programmable aJSON (sampled data for machine learning) [1].

\subsection{Scalable Abstraction for Essential Solutions across Variations}

The web-intensive composite of winCOM promotes digital archives [12] [13] for complex content management in digitally understandable and usable means to enhance human-computer interaction (actionability), engage user experience (contextuality) and assemble brewing aggregation (ubiquity).

Scalable abstraction also denotes scalability as the size of archival documents, which exponentially increased for digital networking service due to growing and increasing demand. Abstraction agility [19] can generalize a solution for multi-problems, and specify the solution to a specific problem by adding specific parts to the problem, as illustrated in Figure 4.

wiseCIO represents the trinity of intelligent cloud service: playable winCOM, programmable aJSON (advanced JSON), and distributed docBases (DdB) with following considerations to scalability [20].

An essential solution across variations - there may be tons of problems, and suggested solutions in reality, which would not help to overcome the complexity that is overwhelming in reality. Scalable abstraction, in a general sense, denotes logical organization and relational grouping categorized as "containers" for easy access, search and assembly such as "shelves," "boxes," "folders," comparable to "situating archives (physical records), designated to hold their very own part of the whole. In a more significant sense, the scalable abstraction allows those archival "containers" to be reused without introducing additional archival containers.

Recursive containers via winCOM-a winCOM can be used as recursive containers via archival transformations. For instance, a winCOM can be seen as a top "shelf", a middle level "box", or a bottom "folder" recursively depending on

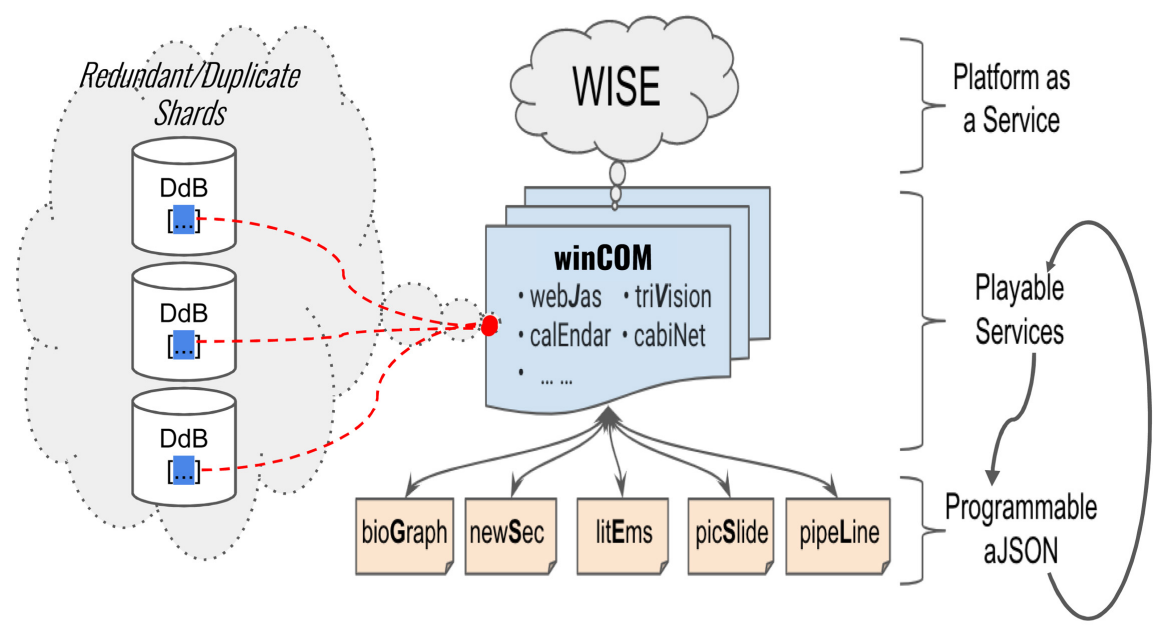

Figure 4. Playable service via hierarchical winCOM and ubiquitous DdB. 
the current context.

Distributed docBases $(D d B)$ - orchestrating winCOM with duplicates and redundancy across clustered servers for agility and scalability in order to grow digital networking service, and manageably increased demand via failover and load-balancing.

A playable service represents a winCOM and the associated archival storage on DdB. a winCOM reflects recursively support for hierarchical extensibility, and the archival DdB promotes analytical synthesis across clustered servers.

\subsection{Testable Algorithms via Step-by-Step Actionable Instructions}

Testable algorithms start in an agile development approach through step-by-step actionable instructions, then become more sophisticated via a test-driven analytical process. The algorithmic practice in favor of experimental "trial \& error" processes via analytical pattern recognition, and recursive 1 earning inference through analytical transformation, which is collaborative and operational on wiseCIO.

Digital archives aim to prepare and present cloud content in many ways by which a computer can understand and make use of [21], so that analytical transformation makes web content useful (actionability), usable (accessibility), and utilizable (applicability). At this point, not everything online is considered as "digitized". For instance, an uploaded PDF document would be considered a "deaf" content without actionable interaction. However machine learning automata can turn web content over DATA from "deaf" into "digitized" [22]. The left side is a traditional PDF document online and the right is digitized via Transformed Analytics without coding required, as illustrated in Figure 5.

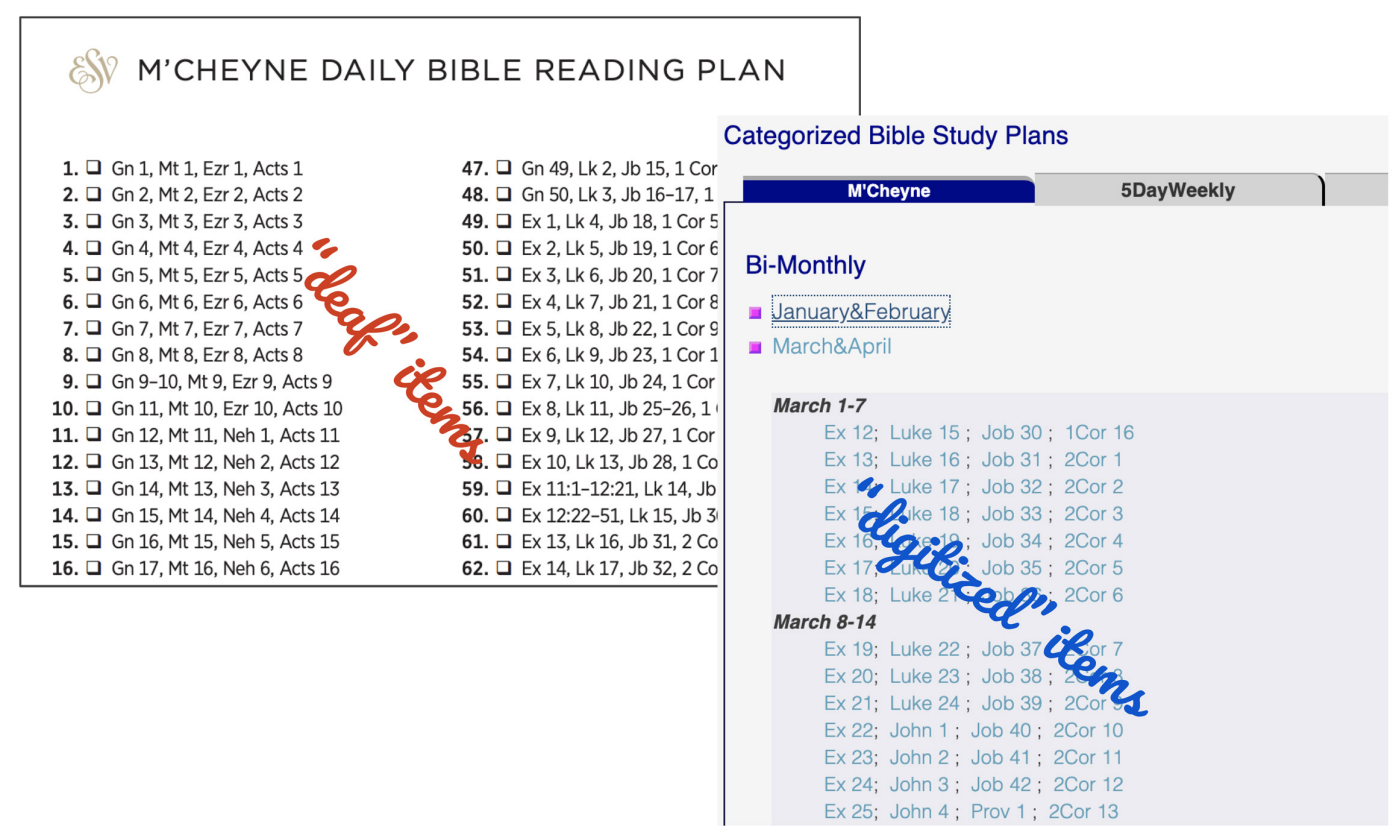

Figure 5. Digital and analytical transformation turn "deaf" into "digitalized" content via machine learning. 
Testable algorithms play a key part in DATA processing for information analytics, patterns recognition, and decision making via machine learning automaton with minimal human intervention. For instance, how to turn a "deaf" web document into "digitized" (Figure 5) would be too trivial to be possible with human invention. In other words, cloud services can be made intelligent to perform specific tasks without using explicit instructions, or relying on patterns and inference [23]. Consequently, machine learning automaton over DATA can automate intelligent UBC processes FAST throughout feasible decomposition, analytical pattern recognition, scalable abstraction and testable algorithms.

\section{3. iDEA for Brewing Aggregation}

iDEA represents intelligence-driven efficient automation over DATA. The intelligent UBC processes collaborate three essential cloud services as a whole universal interface liaising with human-computer interaction, brewing aggregation from ubiquitous DdB (distributed docBases), and centered experience via hierarchical depth and contextual breadth.

iDEA blueprints an intelligent agent known as JIT-MLa that dedicates a series of AAAA tasks to renderable presentation with HCI activated. JIT-MLa stands for just-in-time machine learning automaton, and AAAA for access-assembleaddon-activate and they highly rely on digital archiving, logical inference, and online analytical processing, as illustrated in Figure 6.

Intelligent Brewing Aggregation (IBA), unlike traditional HCI design that supports the major anticipated use cases and situations, enables context-sensitivity that will, according to the context and the user's "will", bring out the context of contextuality over DATA.

\section{1. aCMS: Archival Content Management Service}

Archival Content Management Service (aCMS) aims to discover, exhibit, express

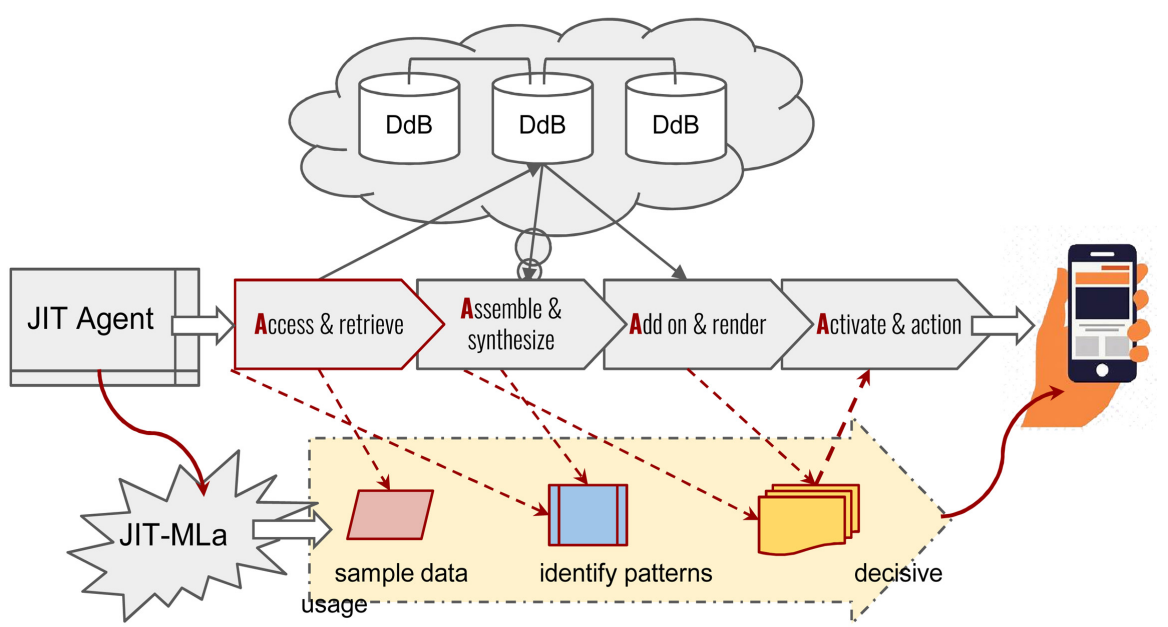

Figure 6. JIT-MLa executes transformational analytics and logical inference over ubiquitous DdB. 
and present well-digitalized archives in the most convenient way based on feasible decomposition. aCMS is embodied as a twin browsing/brewing mechanism in support of both direct accessibility (like traditional webpage) and contextual extensibility as follows:

Direct accessibility-archival digitization is ideally sufficient to view and browse, so a navigating tree is generated and maintained as the user surfs over. Similar to a table of contents, the user can always switch between the current context via the navigating tree to locate and load an individual winCOM that is shareable via a playable command interface (PLI), for instance:

$$
\text { ?cMd=acckey@pathDdB\&params /// RESTful APIs }
$$

where: $c M d$ queries via the access key, operates and interprets the given $\mathrm{DdB}$ accKey should be unique within the same distributed docBase (DdB) params lists Key-Value pairs for possible modification/adjustment

Contextual extensibility based on digital archives, enhances/strengthens analytical transformation via a cloud service - an intelligent agent (JIT-MLa) is responsible to assemble intelligence via brewing aggregation over DATA as an add-on subordinate to the context. By applying context-aware pervasiveness, the access key may be retrieved to assemble winCOMs from ubiquitous DdB, which demonstrates precisely on "brewing" aggregation in support of user-centric experience.

Figure 7 shows you an example of viewing a navigating tree and the brewing aggregation without swapping the current context. The direct accessibility favors rapid locating and easy entrance to individual winCOM (item: R. Nixon) while the contextual extensibility (bars are extensible) is applied to embed content as a whole.

All winCOM over DATA are supportive for contextual extensibility to offer user-centric experience via automated brewing aggregation without coding required. As a highlight with aCMS in particular and wiseCIO in general, the

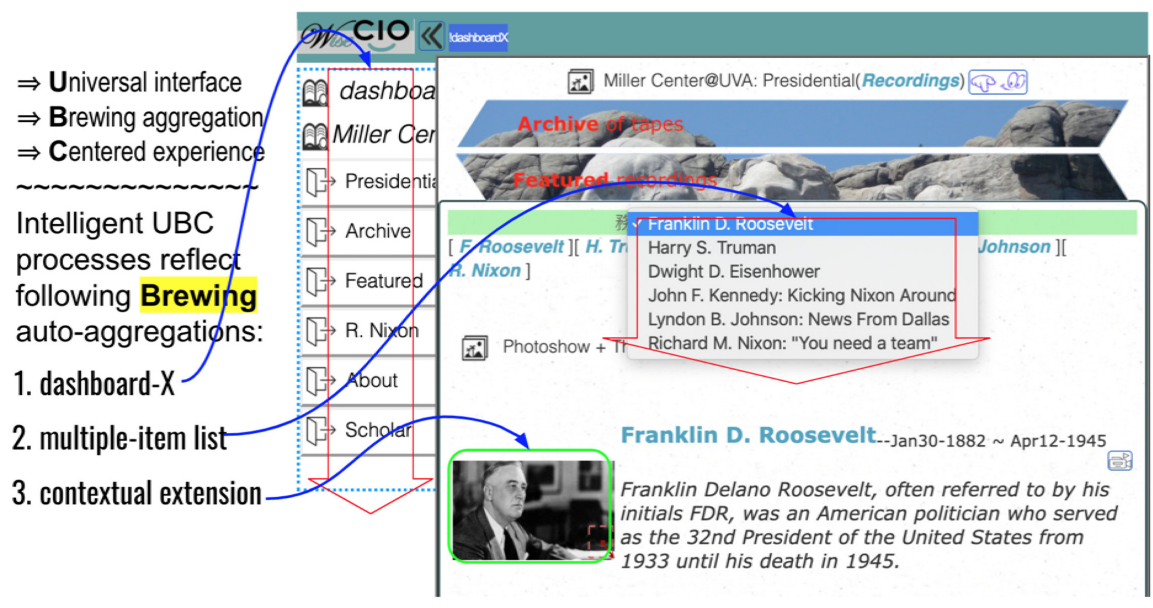

Figure 7. Combinational use of direct accessibility and contextual extensibility. 
brewing aggregation (so different from traditional browsing) means lots of intelligence-driven efficient automation, such as 1) dashboard-X, 2) multi-item list, and 3) contextual extension. Unlike traditional HCI design on major anticipated use cases and situations, the automated brewing aggregation enables context-sensitivity over DATA according to the context and the user's "will".

\subsection{COSA: Context-Oriented Screening Aggregation}

Context-oriented screening [24] was inspired by resume screening a recruiter only spends a few seconds up to 1 minute on viewing an applicant's resume by searching the predetermined keywords according to the job description. If the matching rate is high, the reviewer may initiate consideration to the applicant. The process may fail to find some great applicants, but it is very productive for preliminary screening.

Unlike trying to find any specific contents from a large search engine (Google, Bing, etc.), COSA encourages the user to stay within the subject-based context without swapping pages (websites), then he can discover his interest in the content by searching keywords and viewing the context via the "underlying hyperlinks".

A "underlying hyperlink" means two differences from traditional user experience: firstly, there is no hyperlink anchored onto keywords until he inputs, which would trigger those keywords to anchor the "underlying links"; secondly, the anchored links won't cause context swapping.

Let's take COSA-based courseware [25] as an example, a course learner starts viewing a plain (hyperlink-free) context, so as not to be distracted by extra-unnecessary content. At his first glance, he can briefly view, and quickly figure out whether to explore the content in depth or to skip. If the learner feels that some terminology looks strange, he can input the selected keywords to highlight, then the desired context will be anchored with "underlying links" for him to explore without swapping the current context. This job is usually done by the JIT-MLa that dynamically brews and aggregates content subordinate to the associated keywords, then the user can look via contextual extensibility as illustrated as Figure 8.

COSA creates a proactive experiential scenario for better and user-centric
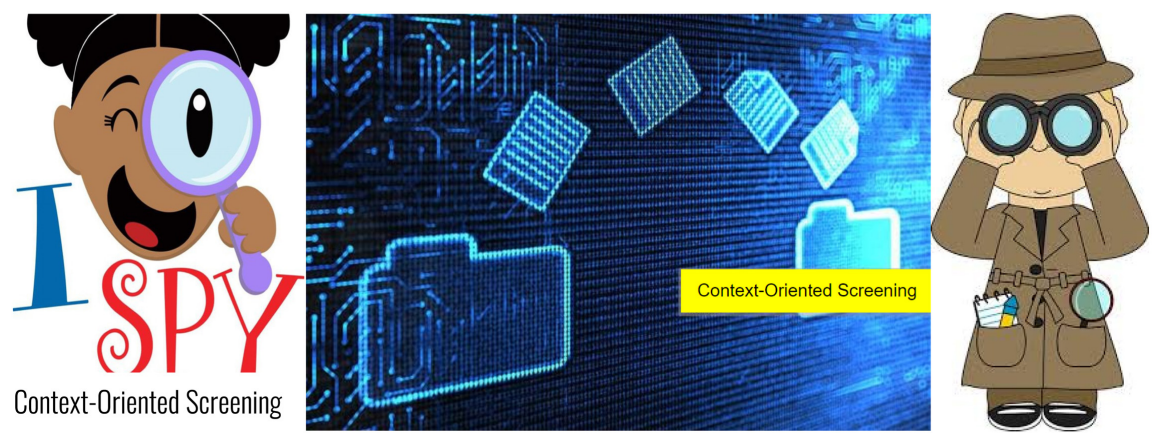

Figure 8. COSA enables proactive brewing aggregation for "zoom-in/out". 
experience: users initially start in brief (without hyperlinks pre-anchored), and wiseCIO enables COSA to discover intelligently and the user to access interested subjects. As it says, "enough is enough", context-aware pervasiveness will offer "fairly good enough" reference triggered by keywords accordingly.

\subsection{DASH: Deliveries Dynamically-Assembled for Search and Hits}

The DASH is sensible to the current context that a user is exploring intentionally or surfing flexibly the managed content (winCOM) and/or manifested content (traditional websites) of his own interest. Acting as a dynamic tracking service via automated brewing aggregation, the sensible DASH assembles the delivered web content at runtime, and then collects its access-keys (similar to URLs) underneath the dashboardX for fast search (what to look) and hits (which to click/hit). This reflectively tracks the user's intention and brings out user-centric experience and engagement through the traceable accessibility.

Managed content on DASH - the managed DASH means winCOM/DATA on wiseCIO that allows the preparer of winCOM to control specific subjects with direct access by planting a potential popups at certain points as the user surfs to the point-the popup won't be popped up until the user gets to the point at runtime. A managed content winCOM typically represents a one-stop-service for well-archived documents to support hierarchical extendibility (extending) and contextual synthesis (shrunk) within the same context. However, the user may also like some traditional browsing experience with page swapping, the managed DASH will meet their needs with "menu-driven browsing" and the menu is treated as dynamic items on DASH.

Manifested content on DASH - the manifested DASH [26] makes wiseCIO as a browser over traditional websites. As a work in progress, the manifested DASH is experimented with chosen websites. It would be a little bit risky if a website is organized ad-hoc, but still worthy while applying more human intelligence for better user experience. The criteria for chosen websites are based on how the website is organized subject to the web expert or machine learning automata because the manifested DASH is a fully automated process. For the sake of convenience, the user has a right to enable or disable both manifested DASH and managed DASH depending on how to use wiseCIO as an excellent browser.

Figure 9 illustrates the use of Harvard University as a sentimental example-the DASH begins with empty items under dash boardX branch, then automated prompts for fast search and hits until web content is open to explore via wiseCIO. In addition to excellent features on wiseCIO including intelligent UBC processing with more automation, the use of wiseCIO together with manifested DASH to explore traditional websites (if chosen) will provide new and better user experience than use of other traditional browsers.

\subsection{OLAS: Online Learning via Analytical Synthesis}

Online Learning via Analytical Synthesis (OLAS) is a twin service to COSA that 


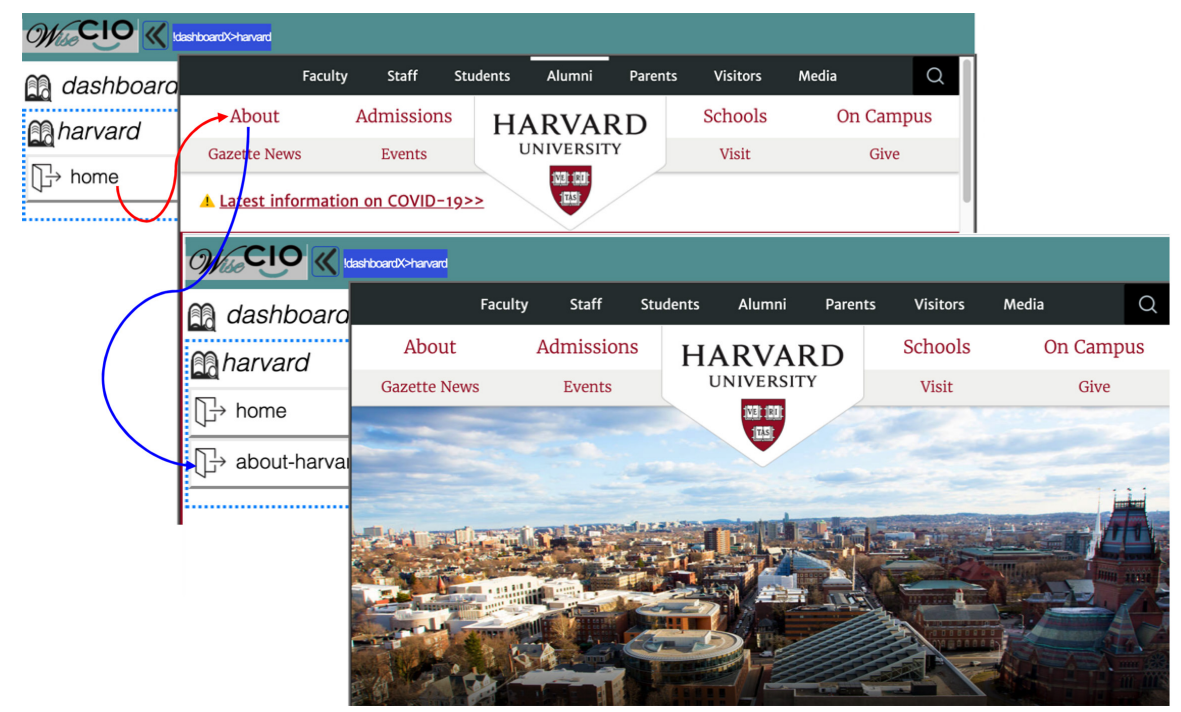

Figure 9. Delivered-assembled for fast search and hits on wiseCIO.

aims to offer users proactive experience, a kind of "Google It" experience, but the user gets what they want within the current context. Cooperatively, OLAS supports COSA by archival transformations with context-aware content reserved through the FAST approach as follows:

Advanced online learning - basic use of the Internet to deliver online courseware at any time, in anywhere via XaaS. According to the eLearning Industry, online education is totally worth the effort because: 1) a learner can learn whatever he wants, 2) self-paced learning, 3) lower cost, 4) comfort and confidence, etc [27]. wiseCIO with its ubiquitous cloud service helps create an advanced online learning environment (AOLE) more friendly (context-oriented), more fostering (pondering and re-thinking), and more fruitful (analytical synthesis) in order to enhance the "readiness to learn without being taught" (Winston Churchill).

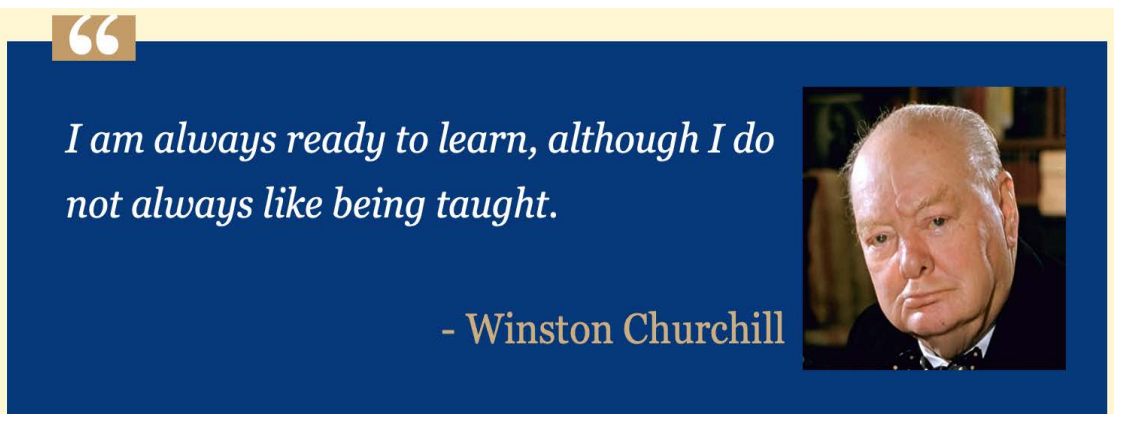

Ubiquitous winCOM-ubiquity is derived from scalability for a holistic organization via digital archives and analytical synthesis over DATA across a cluster of servers. wiseCIO processes ubiquitousness via winCOM for accessibility, availability and actionability over DATA.

Furthermore, the ubiquitous availability means cloud service of CALF- 
cryptography, availability, load-balancing, and failover. A winCOM represents an essential unit of logical organization, and relational document groups by associating multiple shardings of content as a whole, as illustrated in Figure 10.

\subsection{REAP: Rapid Extension to Assembly Presentations}

At the front-end with aCMS, wiseCIO liaises with universal interface to support both direct accessibility and contextual extensibility via winCOM. In addition, wiseCIO with REAP enables ubiquitous winCOM to engage user-centric experience in the back-end.

Viewing from the perspective of big databases, aCMS refers to archived content, and REAP refers to rapid extension for assembled presentations via brewing aggregation. A winCOM is stored as shards in DdB for rapid assembly (transmissible content) and extension (contextual embedment) for active presentations on the client-side, as shown in Figure 11.

According to IBM, big data analytics [28] is the use of advanced analytic techniques against very large, diverse data sets that include structured, semistructured and unstructured data, from different sources. The distributed docBases (DdB) are deployed on a cluster of servers that are integrally-digitalized winCOM with CALF (cryptography, availability, load-balancing, and
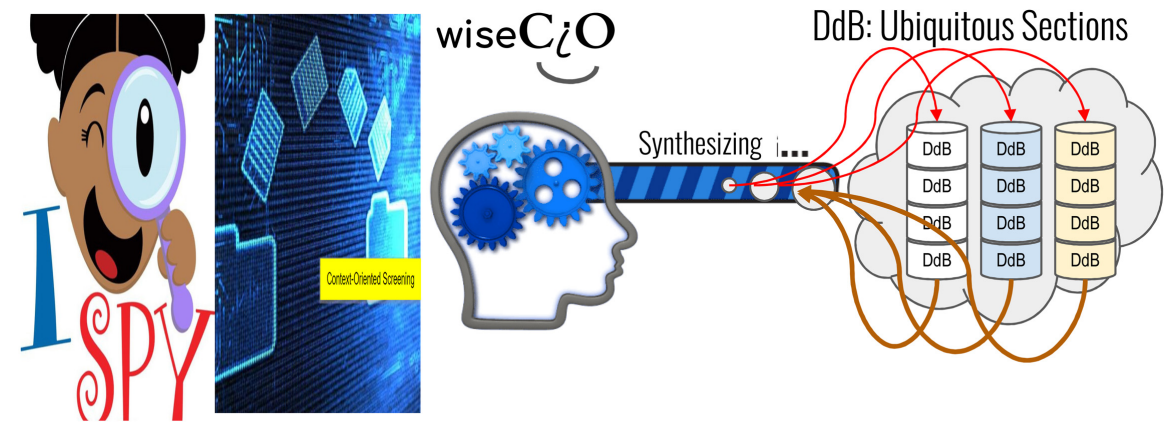

Figure 10. OLAS propagates analytical synthesis over DATA.

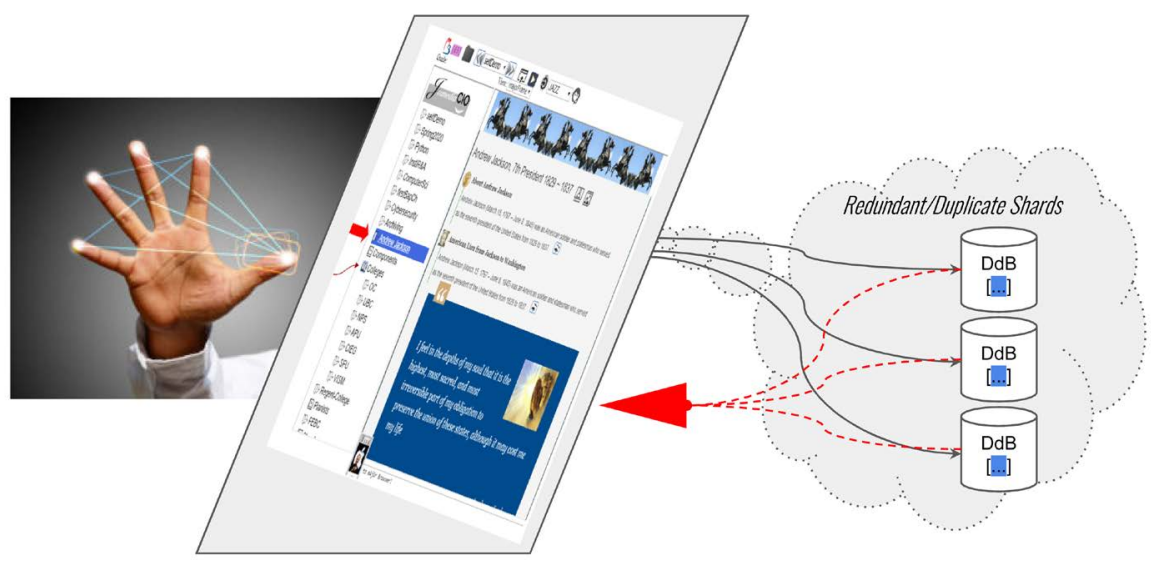

Figure 11. Archival transformations start from actor, through interaction, to assembly. 
failover) features.

\subsection{SPOT: Specific Points on Top (or Hotspot)}

Specific points on top (SPOT) is a graphical user interface that provides at-aglance views of key performance indicators (KPIs) relevant to a particular objective or business process [29] and extends on wiseCIO within the current context for user-centric experience in particular. wiseCIO has two types of SPOT: favorite spot and thematic spot as follows:

Favorite spot-the primary winCOM, like a traditional homepage, will offer a favorite spot whose content (like some snacks) is either manually or automatically updated to help the user find things of common interest at a glance.

Thematic spot-also known as theme-centric spot, a primary winCOM, on the contrary, will also be used as a thematic spot whose content is prepared, presented and published for users to explore relevant things of deep interest at a fast access.

Intelligent brewing aggregation on wiseCIO is open, operational and optimal by enhancing machine learning automaton, with which SPOT can provide an innovative user interface that helps enhance user-centric experience especially for young children and elders with little computer/programming experience, as shown in Figure 12.

According to Figure 12, "one stop service online system" is embodied by SPOT that prioritizes engagement with centered user experience and promotes efficiency and usability of the developed system in use [14]. The SPOT acts like a "key to a drawer"-when "unlocked", the content will be disclosed by embedment without swapping the context, and when "locked", the drawer will be shrunk as "one entry".

The Miller Center established the Presidential Recordings Program (PRP) [30]

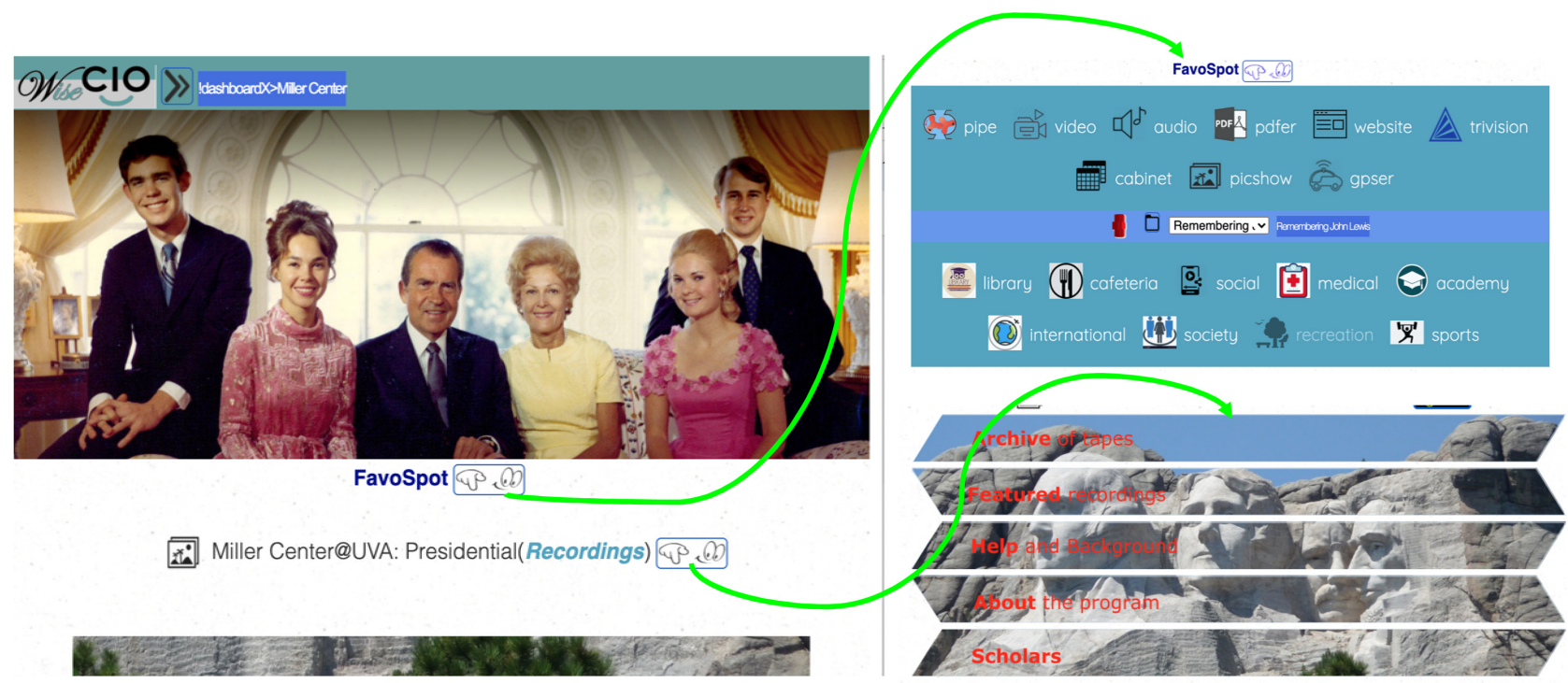

Figure 12. SPOT: Special points on top allow one stop for all (zoom-in/out). 
to make these once-secret White House tapes accessible to all who have an interest or investment in the workings of American democracy. For instance, the PRP has five categories, such as "Archives of tapes", "Featured recordings", "Help and background", "About the program", and "Scholars". As an experiment, the thematic SPOT (Recordings) automates the best practice as a "one stop service online" that betters user experience of accessibility to citizens, journalists, policymakers, scholars, students, teachers with significant ease.

\section{ACTiVE XaaS in Use}

DATA orchestrates wiseCIO in support of XaaS via archival transformations and analytics through intelligent UBC processes. As discussed in previous sections, Computational thinking is applied to integral digitalization via the FAST approach (abstraction \& decomposition, pattern recognition \& algorithms), which provides feasibility and agility for complex and difficult problems to be transformed into a solution.

DATA supports bidirectional approach: top-down analytical (FAST) processing and bottom-up transformational synthesis (brewing aggregation into a larger context) [14], as illustrated in Figure 13.

Case studies in exploring possible and potential XaaS are discussed on a massive basis through ACTiVE mode, that is, accessible/available, contextual and traceable information for vast engagement with managed content for massive deliveries. This section covers efforts to orchestrate ACTiVE XaaS over broad fields.

\subsection{ARM: Archival Repository for Manageable Accessibility}

Archival repository and manageable accessibility (ARM) is very impressive because of wiseCIO's entrance-in-brief (one stop service), and exploration-indepth (contextual extension). As an archivist, I have years of experience managing and categorizing materials in detail, so I am aware of how deep an

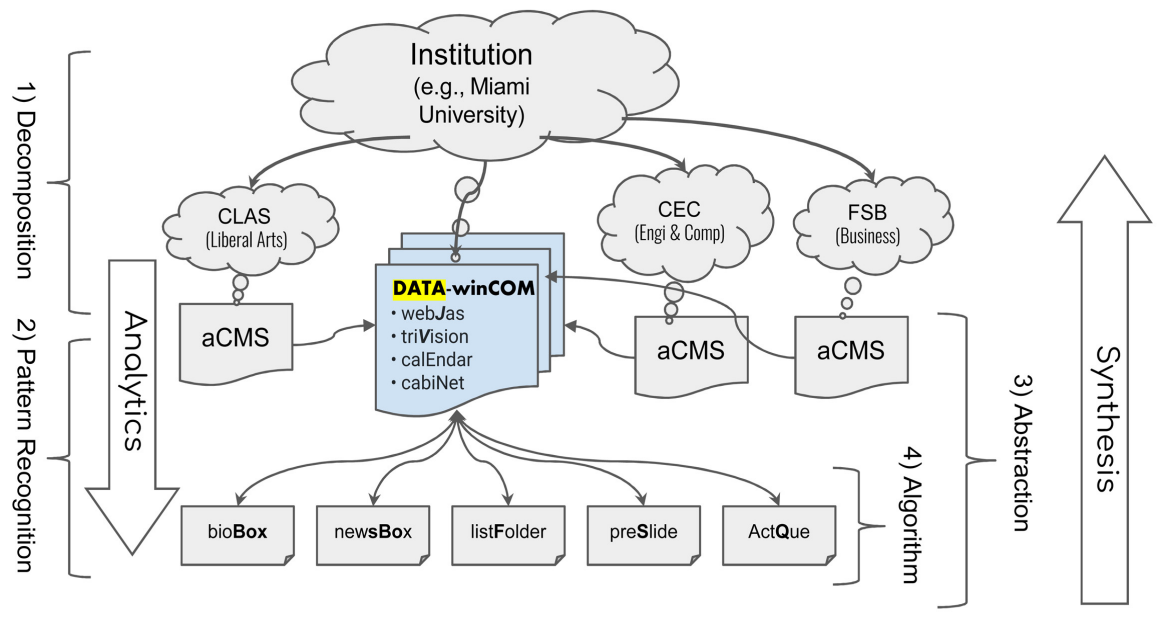

Figure 13. The U-shape approach of digital analytics and archival transformation. 
archival box would be holding organized materials. An archival box will have a detail-oriented index, and may have more than a hundred folders. Each folder may have labels for the identification of its contents, which are authentic certifications and documents. Some of the papers can be very fragile.

Archiving materials in logical organization and relational document groupings by means of physical archiving and so does digital archiving for instance, hierarchical layout via contextual expanding or extension for seventh United States President Andrew Jackson [31]. The capability of a digital archive for historical and biographical exploration allows for varied information groupings congruent to distinct aspects of Jackson's life: early life, military career, political life, and latter years through entrance-in-brief, as shown as Figure 14.

Entrance-in-brief-wiseCIO lays out digital archives in a very brief layout by which a brief entry may take you through a person's whole life within the same context. For instance, six tabs almost cover (through) Andrew Jackseon's whole life from "early life" to "later life" with rich content associated in groupings, such as related video, websites, and so forth.

Explore-in-depth-wiseCIO also supports digital archives through logical organization with considerations to hierarchical depth (as deep as needed) and contextual breadth (as broad as you expect) without forcing the user like "a chasing after webpages". Why? It is all because that hierarchical extensibility allows the user to look into a "drawer" by opening it, and then surf the context by closing the "drawer", which is known as hierarchical extensibility.

In addition, information seekers view primary resource materials and special collections that have been digitized and context-oriented to provide clear documents of Jackson's life relating to Tennessee State history and United States history. This learner-centered approach allows the researcher to explore those topics as needed. Accompanying digital images of primary resources, such as images of his personal correspondence, provide evidence and clarity of information while being highly interesting.

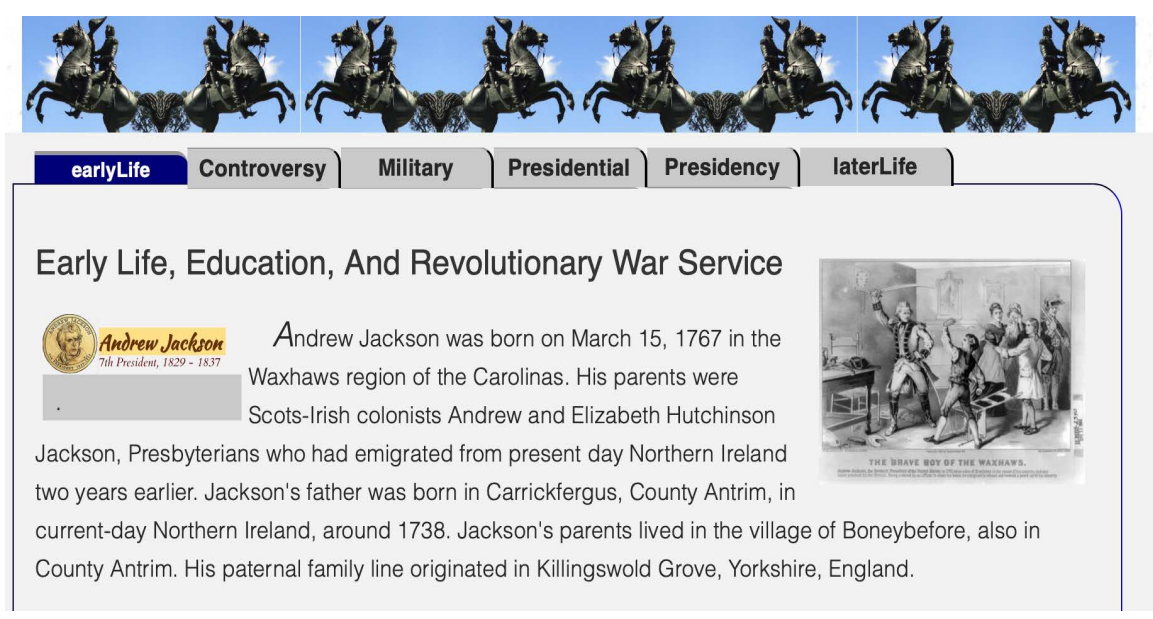

Figure 14. The incredible entrance provides a spectrum through the whole life. 


\subsection{BUS: Biological Understanding from STEM Perspectives}

Computational thinking means not only how we are to think like computers, but also how to make good use of computers via MOLEC (modeling, observing, learning, experimenting and creativity) [32] in support of biological understanding from STEM perspectives.

DATA is promising on how to digitally archive lecture and lab notes, and experimental labs via modeling, observing, (hands-on) learning with creativity promotes better and deeper understanding. Beta lactams are an important class of antibiotics that work by inhibiting cell wall synthesis in bacterial cells. Biochemistry tutorials help STEM students get better understanding visually by using 3D images, and animated GIF, as illustrated in Figure 15.

\subsection{DIGIA: Digital Intelligence Governing Instruction \& Administration}

With the demanding role of school leadership, school administrators often find themselves navigating multiple websites to accomplish tasks needed to support teachers and students. Websites inclusive of the district's student information management system, the state's online teacher evaluation system, and resources to help teachers improve instruction for students are among the many visited by administrators daily. Teachers supported by these administrators also find themselves responsible for managing many online resources to support students as well. However, current websites have all useful information archived in a way that the user has to surf around, which sometimes is quite segmentally distractive.

DATA promises to digitize archives for instruction and administration which have emerged from wiseCIO, to help prepare (well-archived), propagate (synthesis), and present (renderable \& actionable) throughout manageable processing and quick approach. A web-based intelligence service and rich resources
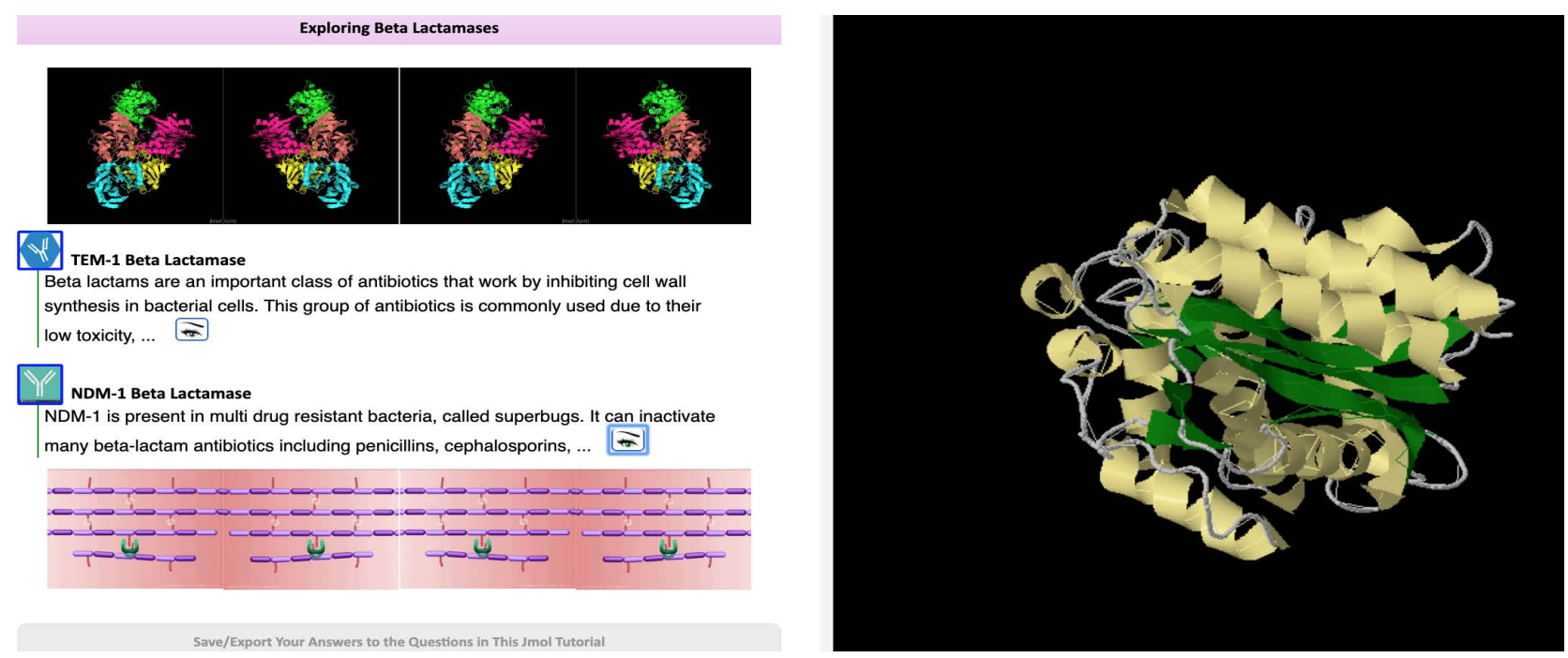

Figure 15. Biological understanding via archived tutorials with Q/A on wiseCIO. 
in the cloud environment will be useful and usable to support the use of digital intelligence to govern both instruction and administration, as illustrated in Figure 16.

Remain focused on primaries - The scarcity of time in today's schools suggests that there is a need for a user-centred context for use, and wiseCIO emerges at digital age to help educators and administrators remain focused on their primary responsibility of instructing students without being distracted or exhausted in the "oceanic browsing". This interactive platform could also be used to engage students with content in an electronic format as well.

School leadership experience-teachers struggle to organize online resources to incorporate technology into lessons on a daily basis. Though there is already a research based established curriculum, often it lacks a daily technology component. However, there remains a need for student engagement. Despite the lack of planning time available to teachers, Herold notes $28 \%$ of principals surveyed thought the integration technologically for all is a transformative method to improve education, and an additional $23 \%$ saw it as a promising idea [33].

Integration of strong curriculum - wiseCIO may help to integrate a well-established strong curriculum into an up-to-date digital platform via a transformatively manageable process that would provide positively profound effects on students' learning. Such a platform would also provide engaging learning opportunities, communication with a network of learners through the internet, innovative ways to assess student learning, and increase in digital fluency. Each of these is necessary skills as students enter the workforce. Additionally, providing students with technological and digital learning experience will also offer equitable learning opportunities for underserved populations [34].

It is the administrator's role to support teachers in the endeavor of integrating technology into their lessons, and it is the district's responsibility to allow autonomy for schools to do so with the understanding that technology based instruction will be paced to support the adopted curriculum. Flexibility of pacing lessons and units should be allowed in order for students to become digitally engaged in such learning.

\subsection{HARP: Historical Archives and Religious Preachings}

As a scholar of religious history and a church historian, I have amassed many

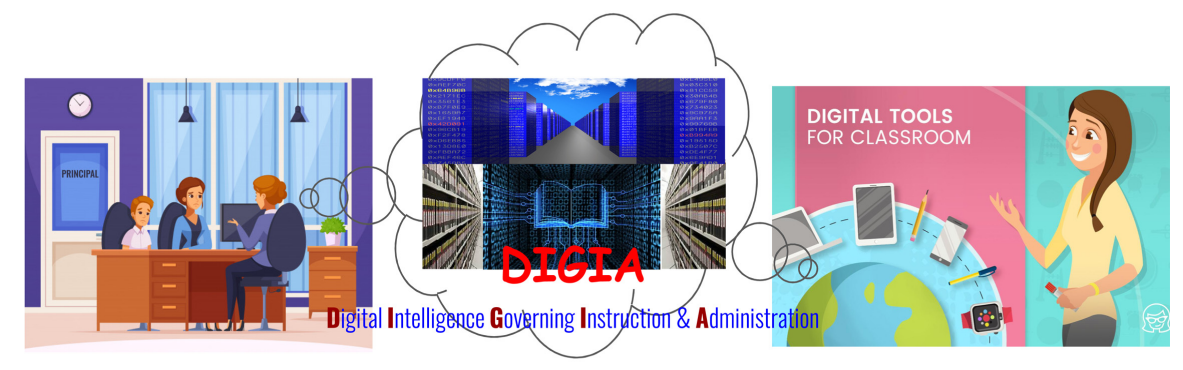

Figure 16. Digital intelligence governing over instruction and administration. 
years of mining and researching at numerous archival sites and collections across the US. The majority of the physical sites have collections that are paper-based or analog-based, but are increasingly moving to include a wider diversity of digital-based resources.

The majority of the physical sites have collections that are paper-based or analog-based, but are increasingly moving to include a wider diversity of digital-based resources.

"no archives, no history" - central to preserving and presenting history, archives are crucial for researching and writing a new denominational history. Transforming paper-based or analog-based collections (physical sites) into a wider diversity of digitized resources holds promise to accessibility and availability in the cloud environment. However many of archives are operating under severe budget and personnel constraints that prevent them from expanding to new digital capabilities that have high value of incorporating emergent digital archiving training and technology.

"digital archives, dicent history"-the CME Church Archival collection in Memphis has identified a wide-range of sources ready for digitization: denominational journals, minutes, reports, pictures, sermons, publications, musical recordings, etc. These sources not only considerably expand the scope of HARP to include more than the collection and preservation of preaching materials but also available, usable and useful online, as illustrated in Figure 17.

DATA promises to incorporate HARP in curricular and pedagogical developments at Lane College, particularly in the newly developed interdisciplinary Religion and Arts track. This track will draw on the disciplines of religion, music, and visual arts, proving both foundational courses and area-specific practicums (e.g., preaching in the religious track). An accessible, interdisciplinary archive of sources/resources for religion, music, and art would be useful for research, teaching, and learning. To enhance the curricular and pedagogical effectiveness of these collaborations, faculty and students alike will need some formal training and digital archiving understanding by use of wiseCIO on digital archives and analytical synthesis across multiple departments, such as the Religion

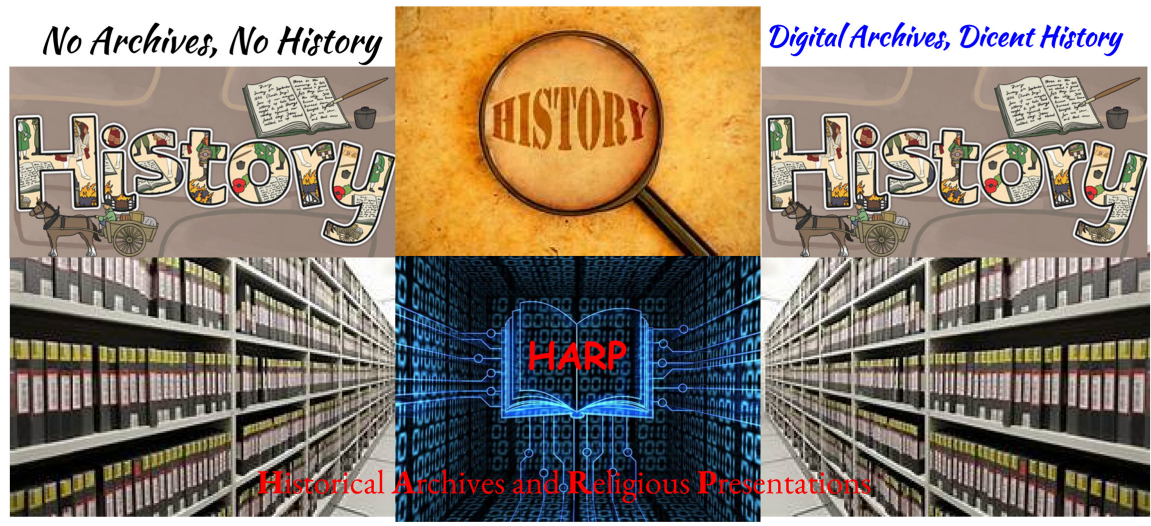

Figure 17. Digital archiving becomes central to a new denominational history. 
and History Departments in HARP collaboration.

\subsection{MATH: Math Application in Teaching and Hands-On Exercises}

In teaching, concrete materials and prompts that are real in students' world of significance help with student's learning. For instance, by making some smiley faces and bringing them to your classroom, you can start with addition, then connect it to multiplication-even if they did not know how to do multiplication, as a last resort, they could count it up, as shown in Figure 18.

Concrete materials - digital materials will greatly signify use of "concrete materials" in teaching via hands-on exercises to a visual, interactive and actionable (VIA) extent that concrete materials can be customized at random. For instance, a girl may like flowers more than smiley faces. By name recognition via machine learning, on the girls screen, the same exercise can have flowers to replace smiley faces. By operating on the VIA materials on the screen acting as a digital "teaching assistant", the teacher may provide students with concrete experiences to help them model, describe, and explore mathematics.

Experimental illustration-addition is essential to multiplication: Use of concrete materials makes learning mathematics experiential so that students can connect multiplication to addition as "repeated addition". They provide students the opportunities to interact with each other, and with the teacher as well.

Hands-on learning is important in the classroom learning experience, so web-based intelligent service helps orchestrate hands-on learning so as to engage students in kinesthetic learning. By operating (dragging and dropping on) visual user interfaces, students may experiment "trial and error", then learn from their mistakes. With wiseCIO the teacher may also create digitally effective learning environments that are valuable to students' prior knowledge. The teacher engages the students in meaningful math lessons and allows students sufficient

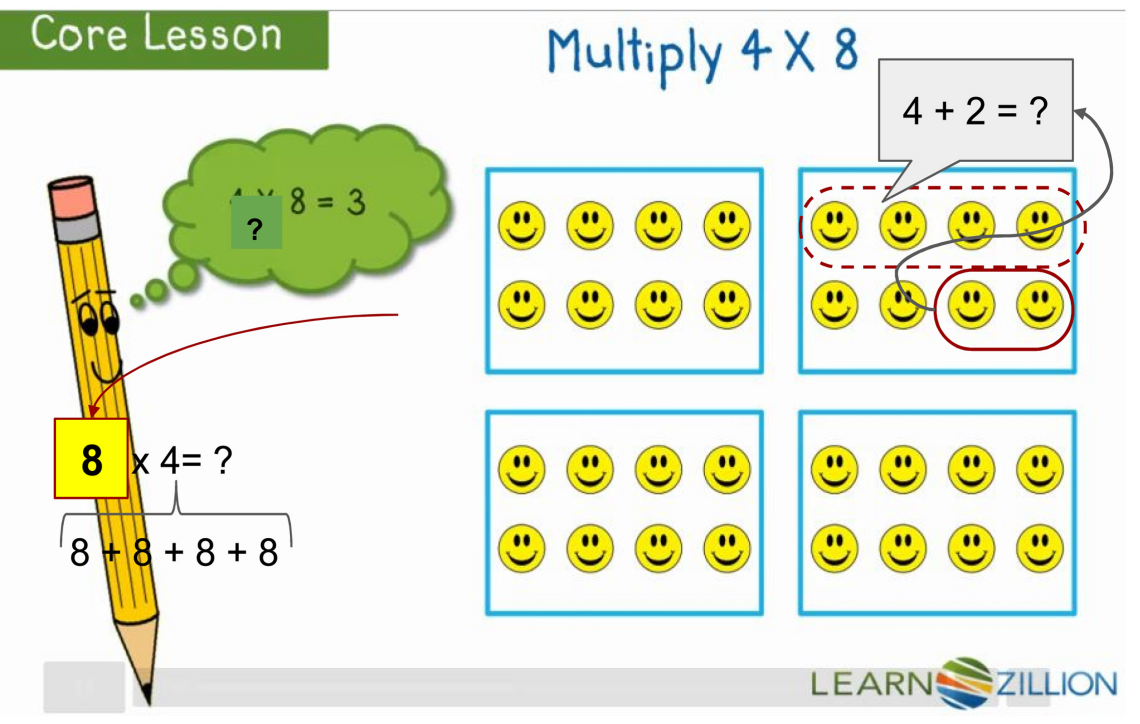

Figure 18. Visual/concrete materials help to see multiplication as "repeated addition". 
time to think about, and discuss problems with their classmates.

wiseCIO will help in dynamical VIA means when students enter school seeing mathematics as an integral part of their world. Students' experiences are, therefore, much more global in essence. They can observe and make links to their prior knowledge when sharing their ideas.

\subsection{SHARE: Study via Hands-On Learning, Assignment and Recap Experience}

wiseCIO allows flexible layouts of often-used icons that provides great convenience via a single block (one-stop service), say "Spring 2020", for instance, there is a set of buttons on the top so that we can conveniently access various online resources, as shown as follows:

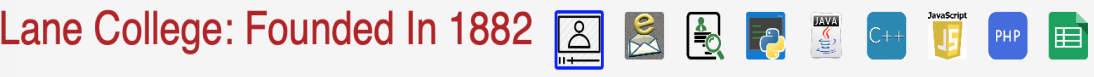

wiseCIO provides excellent hands-on learning experience for students. wiseCIO. On the top, a group of useful tools laid out as buttons, so students can find various online editor for different programming languages such as Python, Java, C++, JavaScript and PHP, etc, which enhances students' hands-on programming experience by comparing and learning multiple languages with understanding of an algorithm. Through hands-on exercises in programming, we can write a program to run and test on the webpage. For instance, through hands-on learning, the professor may teach an algorithm in Python at beginning, then gradually transition to other programming languages such as Java, or $\mathrm{C}++$, etc. As a result, "kill multi-birds with one stone", the students may master multiple languages at the same time.

The online service with wiseCIO is not only those posts by the professor, but also allows us to create our own web session within the profile framework so we students can customize our personal profiles. More significantly, we have flexible ways of submitting our work online, which helps us to keep up with our study progress because they are all online paperlessly.

Timely online sign-in is based on a calendar, and we are required to sign-in in the meantime individually by using computers in the lab. The date and time will be recorded when sign-in; also my photo in display indicates my success in sign-in shown in Figure 19.

Uwiragiye, Claudine, TA.-Teaching Assistant to CSC 131 Claudine Uwiragiye is great and s/he is LOVE: love, objective, victory and enthusiastic; S/he S always had a mind set on going out and making ways for study, family and friends (Video).

Figure 19. The profile acts as an active section allowing paperless submission, online publishing. 
In addition, we can find almost all tools related to our studies within the current course modules, such as BlackBoard, Student/Faculty Portal. Worksheet and Schedule, and we're able to communicate with classmates easily because an email button is there.

\section{Conclusion: DATA in FAST Approach}

This paper presents a central iDEA over DATA that orchestrates wiseCIO for ACTiVE XaaS by UBC process automation via a FAST approach throughout feasible decomposition, analytical pattern recognition, scalable abstraction and testable algorithms. Whose aim is to liaise with human-computer interaction via universal interface, assemble brewing aggregation via online analytical processing and engage centered user exieruece.

What have been achieved-wiseCIO has emerged with an innovative roadmap for intelligent services in the cloud based on aCMS/mCDS (archival Content Management \& Massive Content Delivery Service) and embodied with winCOM, such as COSA (Context-Oriented Screening Aggregator), DASH (Deliveries Assembled for fast Search and Hits), OLAS (Online Learning via Analytical Synthesis), REAP (Rapid Extension \& Active Presentation), and SPOT (Special Points On Top or hotspots for direct accessibility) through brewing aggregation over DATA:

- Digitizing through iDEA-integral digitalization is made through an efficient process automation, representing practical significance via archival transformation and analytics in computational thinking and manageable processing throughout a FAST approach.

- Archiving for aCMS-intensive archive aims at archival content management service that turns digital documents into a variety of labeled categories of such manageability as queryability, assemblability and synthesizability; where a labeled category could be a "shelf", "box", or "folder" for logical organization and relational groupings.

- Transforming via mCDS - transformation of digital archives is to massively deliver high application values as useful and usable intelligence in support of decision-making for Business, Education and Entertainment (iBEE).

- Analytics via winCOM-inline processing of analytics through winCOM is to examine information from distributed docBases using mathematical methods and machine learning techniques to find useful patterns and algorithmic fulfilment for information synthesis.

What are algorithmic via machine learning automata-wiseCIO supports intelligent UBC processes through intelligence-driven elastic automation:

- Universal interface automates LIAiSE (with human-computer interaction) for user interface design through feasible layouts of interactivity and actionability via intelligent systems engineering.

- Brewing aggregation involves archival content management and massive content delivery, instead of simply retrieving, into useful and utilizable intel- 
ligence for business, education and entertainment (iBEE) in support of decision-making.

- Centered experience dedicates ACTiVE XaaS over DATA with accessibility, contextuality and traceability of information for vast engagement with centered user experience in order for efficiency and usability of the developed system in use.

Application scope and limitation-in addition to ACTiVE XaaS in Section 4, wiseCIO has been experimented and applied in broad application scopes, such as "Presidential Recordings Program" (Miller Center, UVA), "Parks Canada" (Canadian National Parks), Coach New York (specializing in luxury accessories), and Remote Online Teaching (Comprehensive Online Teaching \& Learning), esp during the pandemic, which discloses a quite wide application scope. Although cryptography has "by nature" (considered initially) applied to storage and transmission of all digital documents, wiseCIO has not really been used in financial processing that requires high information security so never got experiment-on-attack against CIA (Confidentiality, Integrity, and Availability).

Future plan and further work-wiseCIO, in particular with fully digitized documents in the form of winCOMs, is advantageous to archival management and massive delivery (aCMS/mCDS) through integral \& digital DATA, intelligence-driven automation, and information for vast engagement.

In order to broaden the potential application scope, more effort will be on wiseCIO as an intelligent browser to "brew" traditional websites for better user experience, where integral pattern recognition will play a key role in manifested DASH via machine learning automata.

Universal interfaces liaise with human-computer interaction with great ease. On one hand, intelligence-driven efficient automation is applied with little coding required, but on the other hand, because of the effect of "first buy of customer" (like or dislike), some more deliberate work is needed to beautify the universal interface.

Last but not least, further work will focus more on connectivity and adaptability for enhanced cloud service (XaaS). Connectivity is about a "one stop service" that enables wiseCIO to pull existing websites on the platform so as to be beneficial to user-centric experience; adaptability is about capability of how easy to get existing apps/websites assembled as a part.

\section{Acknowledgements}

This work is partially supported by DOE-MSEIP P120A180072, and our sincere thanks to Ms Evelyn Keele (archivist, TN Room, Jackson-Madison, County Library, Jackson, TN) for ARM (archival repository for manageable accessibility), Dr Melanie Van Stry (Chair of STEM Division at Lane College, Jackson, TN) for BUS (biological understanding from STEM programs), Lance Mak (e-analyst) for DASH (deliveries dynamically-assembled for search and hits), Ms Kimberly N. Quinn (Principal of Denmark Elementary School, Denmark, TN) for DIGIA 
(digital intelligence governing over instruction and administration), Dr. Raymond Sommerville (CME Church Historian / Associate Professor of Religion) for HARP (historical archives and religious preaching and presentations), Dr Peter McCarthy (Associate Professor of Mathematics, Lane College, Jackson, $\mathrm{TN}$ ) for MATH (mathematical applications in teaching and hands-on exercises), Ms. Claudine Uwiragiye (a student in CS, smart and very active as TA in CSC program) for SHARE (studies via hands-on assignments, review and evaluation). Last but not least, I am deeply thankful to Angela for her encouragement, inspiration and loving of wiseCIO (!)

\section{Conflicts of Interest}

The authors declare no conflicts of interest regarding the publication of this paper.

\section{References}

[1] Liang, S., Lebby, K. and McCarthy, P. (2020) wiseCIO: Web-Based Intelligent Services Engaging Cloud Intelligence Outlet. Proceedings of the 2020 Computing Conference, Volume 1, 169-195. https://doi.org/10.1007/978-3-030-52249-0 12

[2] Verma, P. and Kumar, K. (2016) Foundation for XaaS: Service Architecture in 21st Century Enterprise. Service Integration, One Edition. ISBN-13: 978-0692688311. https://www.amazon.com/Foundation-XaaS-Service-Architecture-Enterprise/dp/06 $\underline{92688315}$

[3] Blokdyk, G. (2019) Digitalization through Industrialization a Complete Guide, ISO 26262 a Complete Guide 2019 Edition.

https://www.goodreads.com/book/show/43346236-iso-26262-a-complete-guide---2 019-edition

[4] Jeannette Wing: Computational Thinking. https://www.cs.cmu.edu/ 15110-s13/Wing06-ct.pdf

[5] Denning, P.J. and Tedre, M. (2019) Computational Thinking (MIT Press Essential Knowledge Series). https://doi.org/10.7551/mitpress/11740.001.0001

[6] NSF CSforAll:RPP. https://nsf.gov/pubs/2018/nsf18537/nsf18537.htm

[7] Ameisen, E. (2020) Building Machine Learning Powered Applications: Going from Idea to Product. O’Reilly Media, Newton.

[8] Nilsson, N.J. (1998) Introduction to Machine Learning-An Early Draft of a Proposed Textbook. Robotics Laboratory, Department of Computer Science, Stanford University, Stanford. https://ai.stanford.edu/ nilsson/MLBOOK.pdf

[9] SAS Insights: Machine Learning. https://www.sas.com/en us/insights/analytics/machine-learning.html

[10] Srivastav, M.K. and Nath, A. (2016) Web Content Management System. International Journal of Innovative Research in Advanced Engineering, 3, 51. https://www.researchgate.net/publication/299438184 WEB CONTENT MANAGE MENT SYSTEM

[11] Blokdyk, G. (2019) Database Integrity A Complete Guide, 2020 Edition. 5STARCooks, Toronto, ON, Canada.

https://www.kobo.com/ca/en/ebook/data-integrity-a-complete-guide-2020-edition 
[12] Hodge, G.M. (2000) Best Practices for Digital Archiving-An Information Life Cycle Approach. D-Lib Magazine, 6. http://www.dlib.org/dlib/january00/01hodge.html

[13] Archives @ PAMA, Region of Peel: How Do Archivists Organize Collections? https://peelarchivesblog.com/2015/08/26/how-do-archivists-organize-collections

[14] Sriarunrasmee, J. and Anutariya, C. (2020) The Development of One Stop Service Online System based on User Experience Design and AGILE Method. The 11 th International Conference on E-Education, E-Business, E-Management, and E-Learning, Osaka, 10-12 January 2020, 64.

https://www.researchgate.net/publication/341143924 The Development of One S top Service Online System based on User Experience Design and AGILE Met $\underline{\text { hod }}$

[15] Sriram, R.D. (1997) Intelligent Systems for Engineering-A Knowledge-Based Approach. https://www.springer.com/gp/book/9783540761280

[16] Nakano, R. (2001) Web Content Management: A Collaborative Approach. https://www.amazon.com/Web-Content-Management-Collaborative-Approach/dp/ $\underline{0201657821}$

[17] Mishra, D.D. (2018) Divide and Conquer Paradigm. https://www.includehelp.com/algorithms/divide-and-conquer-paradigm.aspx

[18] Gamma, E., Helm, R., Johnson, R. and Vlissides, J. (1994) Design Patterns: Elements of Reusable Object-Oriented Software.

https://www.amazon.com/Design-Patterns-Elements-Reusable-Object-Oriented/dp $\underline{10201633612}$

[19] Aucsmith, D. (1998) Information Hiding, Second International Workshop, Portland, Oregon, USA. Proceedings. 14-17 April 1998, Berlin.

[20] Liang, S., Puette, J. and Lu, Q. (2004) Quantifiable Software Architecture of Dependable Systems of Systems. In: de Lemos, R., Ed., Architecture, Dependable Systems II, Springer Verlag, Berlin, 241-265. https://doi.org/10.1007/978-3-540-25939-8 11

[21] Dobreva, M. and Ivacs, G. (2017) Digital Archives: Management, Use and Access. Cambridge University Press, Cambridge.

[22] McCarthy, J. (2020) Automata: Compiling State Machines. https://docs.racket-lang.org/automata/index.html

[23] Kundan, A.P. (2019) Intelligent Automation with VMware: Apply Machine Learning Techniques to VMware Virtualization and Networking. Packt Publishing, Birmingham.

[24] Interaction Design Foundation-Context-Aware Computing. https://www.interaction-design.org/literature/book/the-encyclopedia-of-human-co $\underline{\mathrm{mpu}-}$ ter-interaction-2nd-ed/context-aware-computing-context-awareness-context-aware -user-interfaces-and-implicit-interaction

[25] Liang, S. (2018) COC: Web-Based Intelligent Services Enabled Comprehensive Online Courseware. CUR Biennial Conference, Crystal City, 30 June-3 July 2018.

[26] Manifested DASH Has Chosen Harvard University. https://www.harvard.edu

[27] Norman, S. (2016) 5 Advantages of Online Learning: Education without Leaving Home. eLearning Industry, March 10, 2016.

https://elearningindustry.com/5-advantages-of-online-learning-education-without-1 eaving-home 
[28] IBM. What Is Big Data Analytics? https://www.ibm.com/analytics/hadoop/big-data-analytics

[29] Few, S. (2009) Information Dashboard Design-Effective Visual Communication of Data. O’Reilly Media, Newton.

[30] The Miller Center Established PRP in 1998. Between 1940 and 1973, Six Consecutive American Presidents Secretly Taped Thousands of Their Meeting and Telephone Conversations.

https://millercenter.org/the-presidency/secret-white-house-tapes

[31] Tennessee Virtual Archive.

https://teva.contentdm.oclc.org/digital/search/searchterm/Andrew\%20Jackson

[32] Biomolecules.

https://www.biologydiscussion.com/biomolecules/biomolecules-top-4-classes-of-bi omolecules/11169

[33] Herold, B. (2018) What Principals Really Think about Tech. Education Week. https://www.edweek.org/ew/articles/2018/04/18/what-principals-really-think-about -tech.html

[34] Whitehead, B., Jenson, D. and Boschee, F. (2002) Planning for Technology: A Guide for School Administrators, Technology Coordinators, and Curriculum Leaders. Corwin Press, California. 